\author{
Matthew Santer ${ }^{1}$ \\ Lecturer in Aerostructures \\ Department of Aeronautics, \\ Imperial College London \\ Prince Consort Road, \\ London, SW7 2AZ, UK \\ e-mail: m.santer@imperial.ac.uk \\ Sergio Pellegrino \\ Joyce and Kent Kresa Professor of Aeronautics \\ and Professor of Civil Engineering \\ California Institute of Technology, \\ 1200 E. California Boulevard, MC 301-46, \\ Pasadena, CA 91125 \\ e-mail: sergiop@caltech.edu
}

\section{Concept and Design of a Multistable Plate Structure}

A concept is presented for a compliant plate structure that deforms elastically into a variety of cylindrical shapes and is able to maintain such shapes due to the presence of bistable components within the structure. The whole structure may be fabricated as a monolithic entity using low-cost manufacturing techniques such as injection molding. The key steps in the analysis of this novel concept are presented, and a functional model is designed and constructed to demonstrate the concept and validate the analysis. [DOI: $10.1115 / 1.4004459]$

\section{Introduction}

Imparting a smooth curvature change to a surface has applications to, for example, flow control methodologies [1], active jet inlets, and adaptive, reconfigurable reflector surfaces. Space is also a particularly suitable environment for compliant mechanisms, due to limitations on power availability and the difficulty of maintaining adequate lubrication when conventional mechanism components are used repeatedly.

This paper presents a novel concept for a plate structure that can take up a variety of cylindrically curved shapes [2]; this proposed concept is realized as a single-piece compliant mechanism. The structure is multistable, which means that it has several different configurations of stable equilibrium without any external loads and, hence, can hold these shapes without the need for continued actuation. This inherent multistability is one of the primary advantages of the proposed structure over alternative concepts for morphing surfaces. Multistability may be achieved by material selection, for example, by exploiting anisotropy [3,4] or by snapthrough behavior [5-7]. It is this approach that is adopted here; the proposed solution provides a wider range of alternative configurations than previously proposed surfacelike multistable structures.

The combination of mechanisms-both conventional and compliant-with a continuous structure to achieve large deformations via distributed compliance has been the subject of much research. For example, topological optimization techniques have been proposed to determine the compliant substructure that enables the continuous morphing of an airfoil leading edge with compliant skin $[8,9]$ and an adaptive antenna structure [10]. An alternative concept proposed for morphing an airfoil trailing edge involves the localized replacement of the wing skin with compliant bistable composite plates [11]. This is a promising approach, but the achieved deformation is restricted by the geometrically stable configurations of the bistable plates.

The concept that is presented in this paper was inspired by the adaptive truss proposed in Ref. [7] in which the members forming the three main longerons are replaced with linear bistable elements. This results in a reconfigurable multistable structure with $2^{n}$ discrete stable states, where $n$ is the number of bistable actuators. The concept that is utilized is that of binary robotics, in which a continuous motion is approximated by actuating a large number of discrete actuating elements [12]. An adaptive plate based on double layer Kagome lattice structures was presented in Refs. [13] and [14]. In these structures, two flat face-sheets, each consisting of a lattice of structural members, are held at a distance by a support structure consisting of slender structural members,

\footnotetext{
${ }^{1}$ Corresponding author.

Manuscript received November 25, 2010; final manuscript received June 17 , 2011; published online July 27, 2011. Assoc. Editor: Nancy Johnson.
}

thus forming a kind of sandwich plate structure. Curvature is imparted to this plate by extending or contracting a number of actuators. Instead, our proposed concept achieves multistability through the chaining of discrete bistable components that are geometrically offset from a thin plate ("compliant plate") by means of a series of inclined thin plates ("corrugated plate structure"). Changing the length of a bistable component has the effect of bending the plate, as shown in Fig. 1.

The advantage and novelty of this approach are that it enables the benefits of multistability - in particular, the ability to lock in a deformed configuration without the need for continued actuation-to be combined with the benefits of morphing plates. The replacement of the internal truss structure, such as that adopted in Refs. [13] and [14], with corrugated webs simplifies the fabrication of the multistable plate structure. As will be shown, this enables the structure to be constructed as a single monolithic entity. The approach pursued in this paper is aimed at applications that require a range of cylindrical configurations only. If a wider range of shapes were required, for example, to include also doubly curved (nonzero Gaussian curvature) configurations, then the double-layer Kagome lattice in Ref. [13] would be a better structural architecture to start from. Bistable actuation could then be incorporated in such architecture following the same approach presented here.

The paper is arranged as follows. In Sec. 2, the concept is discussed in a general form, and a simple proof-of-concept model that demonstrates its feasibility is presented. In the following section, a simple analysis determines the required characteristics of the force-displacement relationship of bistable elements, which are able to hold a given compliant plate in a deformed configuration. Section 4 presents a suitable choice for the bistable element and Sec. 5 presents the design of a functional prototype that has been fabricated using rapid prototyping technology. This structure contains a chain of five bistable elements. Section 6 concludes the paper and suggests a number of areas for further work.

\section{Structural Concept}

A structure with the following properties is desired:

- It should have (i) a flat stable configuration, (ii) a uniformly curved, cylindrical stable configuration, and (iii) additional, intermediate stable configurations that consist of flat and uniformly curved regions.

- Once the structure has been set into one of its stable configurations, it should maintain this configuration for at least a specified time duration, if any external disturbances are smaller than a specified magnitude.

- It should be scalable.

The reason why the range of required shapes for the structure has been restricted to singly curved is to avoid the much larger 


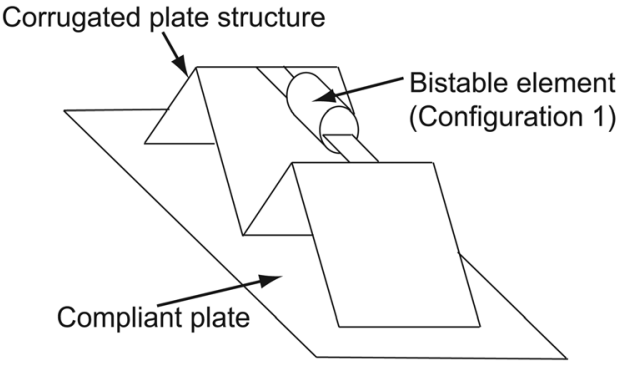

(a)

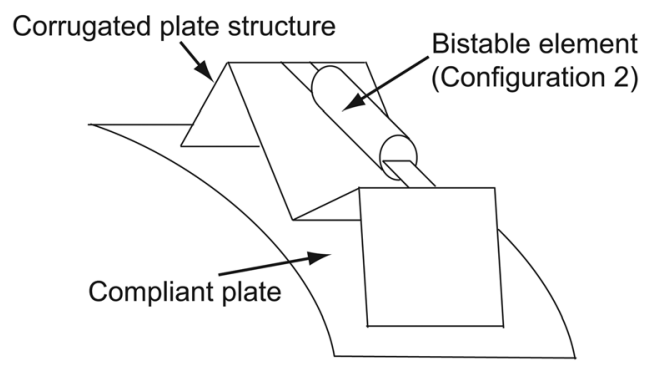

(b)

Fig. 1 Schematic diagram of structural concept: (a) flat plate and bistable element in configuration 1; (b) curved plate and bistable element in configuration 2

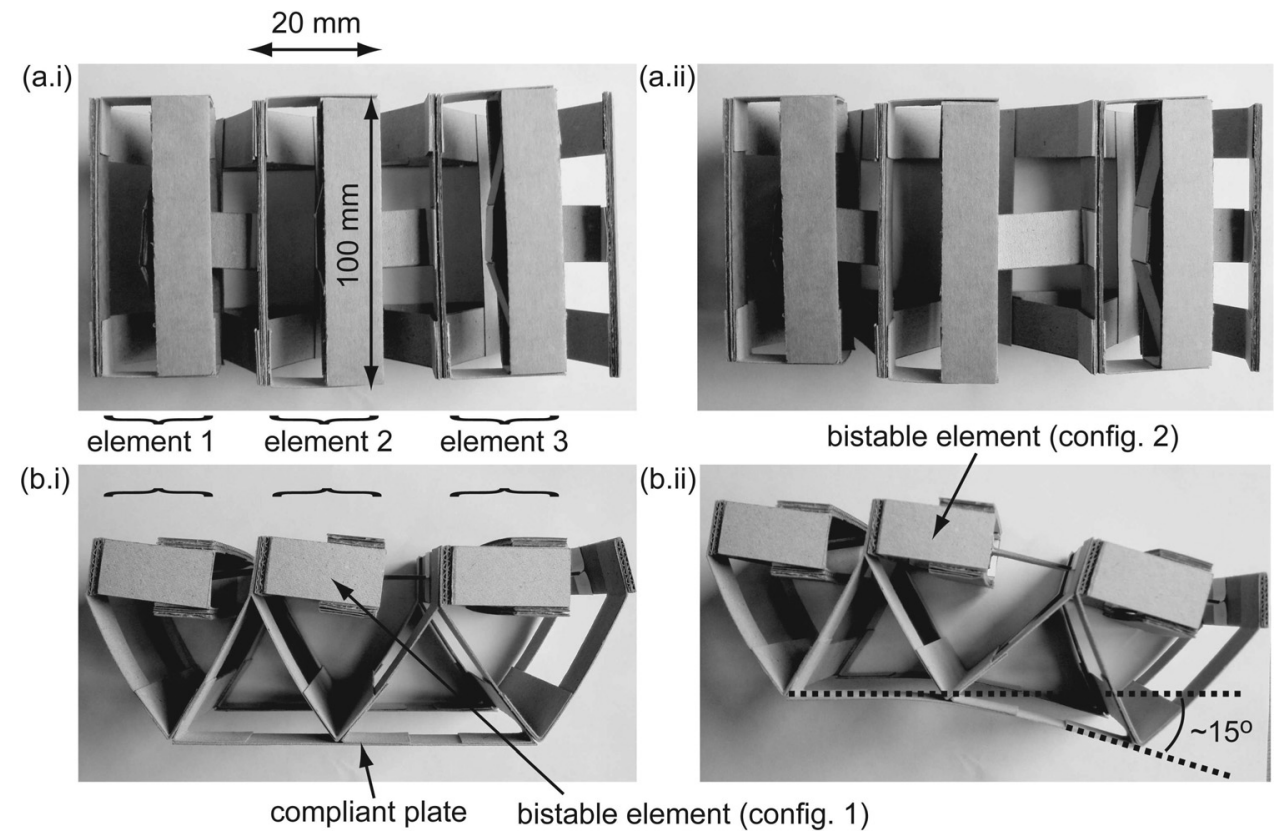

Fig. 2 Cardboard proof-of-concept model in (a) initial configuration: (i) top view and (ii) side view; (b) deformed configuration: (i) top view and (ii) side view

forces needed to stretch the compliant plate. In future, it may be desirable to extend the present work to shape changes between arbitrary curvatures, including nonzero Gaussian curvatures. The second point is to account for initially stable configurations losing their stability due to time-dependent material behavior such as viscoelasticity.

A simple proof-of-concept model made of cardboard is shown in Fig. 2. This structure consists of three adjacent units forming equilateral triangles with a side length of $\sim 100 \mathrm{~mm}$. Each bistable element consists of a pair of cardboard plates forming a snapthrough truss, based on the concept adopted by Schiøler and Pellegrino [7]. All connections between the plates are made with thin sheets of paper. The effect of actuating the central bistable component is shown in Figs. 2 (a.ii) and (b.ii). It can be seen that the structure bends by $15 \mathrm{deg}$ and remains curved when the actuation force is removed. It is thus clear that the concept is viable, and so a more detailed design is developed.

\section{Analysis of Actuation Force}

A key step in the realization of the proposed concept is to determine the minimum force that has to be resisted by the bistable elements in order to hold the plate structure in a curved configuration. It will be assumed that the structure is stress free when it is flat.
A simplified two-dimensional analysis can be carried out on a single bistable element connected to a compliant plate by means of a regular pin-jointed triangular truss, Fig. 3(a). When the bistable element is actuated, its length increases by a known amount, Fig. 3(b). Although the proposed concept requires a chain of actuating elements, the three adjacent units shown in the figure fully capture the behavior of the whole structure. This is because the curvature imparted to the plate by a single bistable element is localized to the region under the element. The effects of actuating multiple elements may be determined from superposition.

In Fig. 3(a), the structure is shown with the compliant plate in the flat configuration. In this configuration, all of the isosceles triangles forming the truss are identical; two sides have length $a$ and the third side has length $L$. This length is the initial, i.e., unactuated, length of the bistable element, and has been represented by a filled rectangle. The internal angles are $\theta_{1}$ and $2 \phi_{1}$, as shown. The thick line at the bottom represents the compliant plate. For clarity and to emphasize that the compliant plate is continuous, a small offset has been shown between the plate and the joints of the truss; however, the hinges and the plate are assumed to be collinear in the analysis.

The bending stiffness of the connections between the members of the truss is assumed to be negligible in comparison to that of the plate; if it is not negligible, then springs could be incorporated into the model shown in Fig. 3 to form a pseudorigid-body 


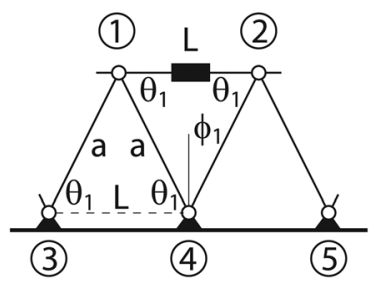

(a)

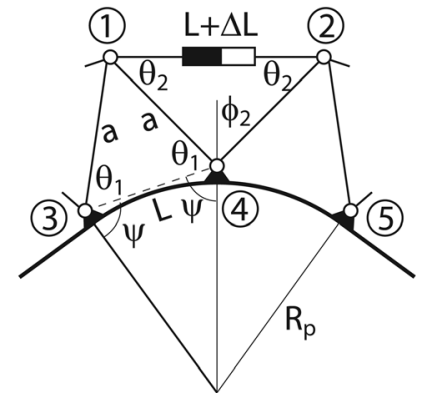

(b)
Fig. 3 Two-dimensional geometric model of the multistable plate structure concept

representation of the compliant mechanism [15]. This refinement will not be considered in the present analysis.

When the length of the bistable element connecting hinges 1 and 2 is increased by $\Delta L$, represented by the hollow rectangle in Fig. 3(b), the compliant plate bends to accommodate this change. It can be assumed that when an actuator in a single triangle changes length, all other triangular units in the structure are substantially unaffected, i.e., in Fig. 3(b), the distance between hinges 3 and 4 is still approximately equal to $L$. Hence, the effect of actuating the bistable element is to change the internal angles of its associated triangular element to $\theta_{2}$ and $2 \phi_{2}$, but the angles in the neighboring triangles remain $\theta_{1}$ and $2 \phi_{1}$.

The angles $\theta_{1}, \phi_{1}, \phi_{2}$, and $\psi$, defined in Fig. 3, have the expressions

$$
\begin{gathered}
\theta_{1}=\arccos (L / 2 a) \\
\phi_{1}=\left(\pi-2 \theta_{1}\right) / 2 \\
\phi_{2}=\arcsin ((L+\Delta L) / 2 a) \\
\psi=\pi-\theta_{1}-\phi_{2}
\end{gathered}
$$

The compliant plate does not kink at a single point but instead bends to accommodate the rotation of the two outer triangular units. This deformation can be analyzed by considering the forces transmitted between the truss and the compliant plate, as seen in the free body diagrams shown in Fig. 4. Assuming small deflections, the analysis can be carried out in the undeformed configuration.

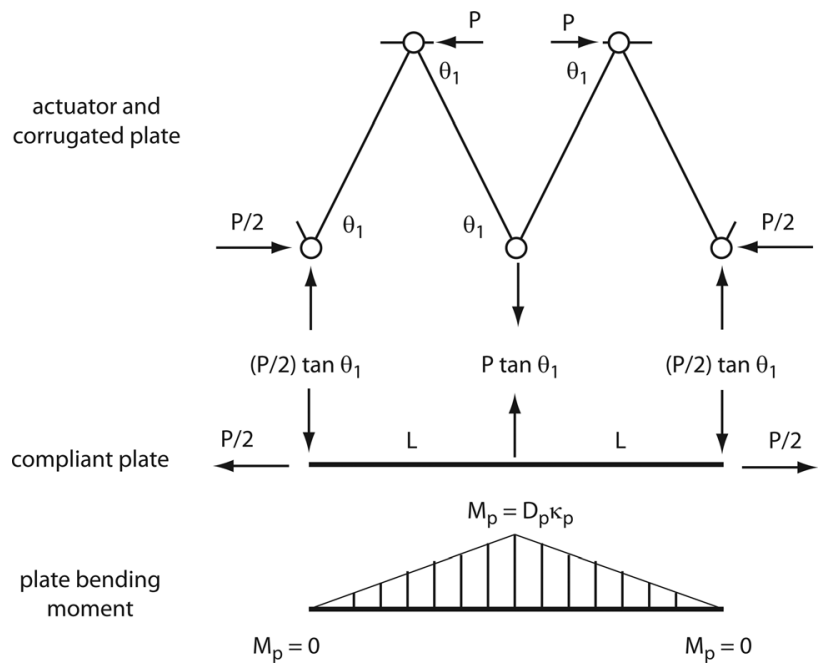

Fig. 4 Free body diagrams and associated bending moment in the plate when a single bistable element is actuated
It can be seen that the effect of a force $P$ in an actuated bistable element is to place a region of the compliant plate into combined tension and three-point bending, with the bending moment distribution shown at the bottom of Fig. 4. Note that the maximum value, $M_{p}$, is reached at the midpoint

$$
M_{p}=D_{p} \kappa_{p}=\frac{P L}{2} \tan \theta_{1}
$$

in which $\kappa_{p}$ is the curvature at the midpoint of the plate, and $D_{p}$ is the bending stiffness of the plate (assumed to have unit width)

$$
D_{p}=\frac{E t^{3}}{12\left(1-\nu^{2}\right)}
$$

multiplied by the width of the plate. $E$ and $\nu$ are the modulus and Poisson's ratio of the compliant plate, respectively, and $t$ its thickness.

For the structure to be able to remain locked in this deformed configuration, the force at which the bistable element snaps must be greater than the value of $P$ obtained from Eq. (5). The plate curvature $\kappa_{p}$ in Eq. (5) can be estimated by initially considering a uniform curvature over the region of plate of length $2 L$. With this assumption, one can draw a circular arc through hinges 3,4 , and 5 in Fig. 3(b); the radius of this circle is

$$
R_{p}=\frac{L}{\pi-2 \psi}
$$

and substituting Eq. (4), the corresponding uniform curvature is given by

$$
\overline{\kappa_{p}}=\frac{1}{R_{p}}=\frac{\pi-2 \psi}{L}=\frac{2\left(\theta_{1}+\phi_{2}\right)-\pi}{L}
$$

The actual curvature of the plate has a bilinear form with a maximum value $\kappa_{p}$ at the center. Because the integral of the curvature along the plate must be the same, the peak curvature, $\kappa_{p}$, is given by

$$
\begin{aligned}
\kappa_{p} & =2 \overline{\kappa_{p}}=\frac{4\left(\theta_{1}+\phi_{2}\right)-2 \pi}{L} \\
& =\frac{4(\arccos (L / 2 a)+\arcsin ((L+\Delta L) / 2 a))-2 \pi}{L}
\end{aligned}
$$

Then, the force $P_{\min }$ that must be carried by the bistable element without snapping, in order for a plate of unit width to remain locked in the curved configuration, is

$$
P_{\min } \geq 4 \frac{D_{p}\left(2\left(\theta_{1}+\phi_{2}\right)-\pi\right)}{L^{2} \tan \theta_{1}}
$$

and, substituting Eqs. (1) and (3)

$$
P_{\min } \geq 4 \frac{D_{p}(2(\arccos (L / 2 a)+\arcsin ((L+\Delta L) / 2 a))-\pi)}{L^{2} \tan \theta_{1}}
$$

The effect of actuating additional bistable elements in the chain may be determined by superposition of the corresponding free body diagrams. It has been shown in Fig. 4 that actuating a single bistable element results in a bilinear bending moment distribution in the compliant plate immediately below that element. When an adjacent element is actuated, it generates an identical bending moment distribution but shifted along by one unit. Superposing both diagrams results in a region of constant bending moment, and hence actuating multiple elements results in regions of uniform curvature, but the minimum required force in each element remains that determined in Eq. (10). It can be shown by 


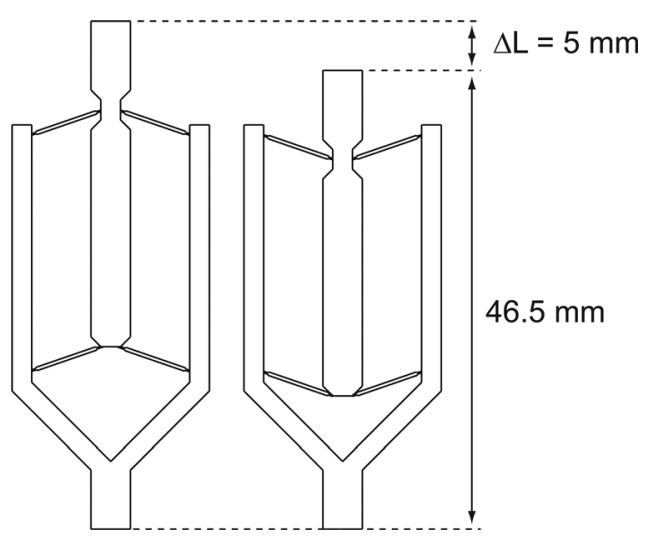

Fig. 5 Design concept of snap-through strut with stroke of $\approx 5 \mathrm{~mm}$ (from Ref. [7])

considering joint equilibrium that the two outer diagonal members are subject to equal compressive forces and the two inner diagonal members to tensile forces of the same magnitude. When adjacent segments of plate are also curved, by superposition, the forces in all inner diagonal members become zero.

Although the above analysis is idealized, its simple parameterization allows a rapid and full exploration of the design space and enables us to evaluate designs that can be realized within the limitations of available materials and manufacturing technologies. This obviates the need to run many lengthy nonlinear finite element analyses. As mentioned above, the assumption is made that the bending stiffness along the edges of the corrugated plate is negligible in comparison to that of the compliant plate; this is a reasonable assumption since our objective is to develop designs in which these connections are thin. The assumption that the analysis of the forces and moments in the structure can be done in the original, i.e., undeformed configuration, is not considered as a significant limitation because, in practice, the geometric distortion of each individual repeating element is small. Of course, the cumulative shape change resulting from actuating multiple adjacent bistable elements is large. An advantage of the simplified approach that has been adopted is that it led to the insight that actuation of multiple bistable elements results in zero force in all the diagonal members.

\section{Bistable Element}

The analysis in Sec. 3 incorporates the bistable element as an idealized component that imparts a change in length and resists an

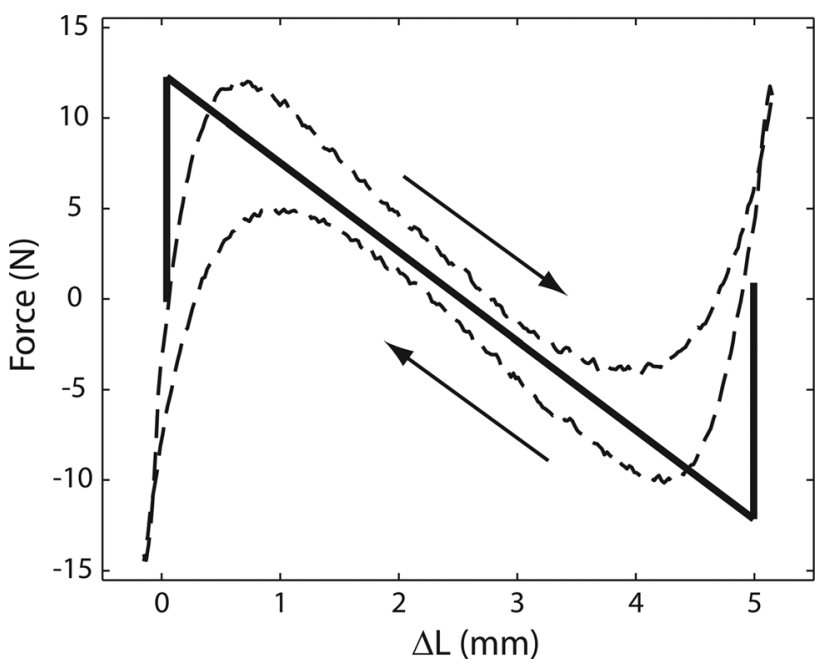

Fig. 6 Elastic prediction versus measured behavior of snapthrough strut (adapted from Ref. [7])

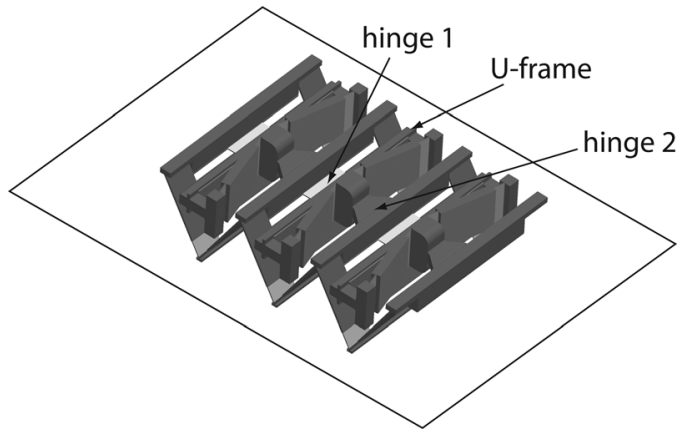

Fig. 7 Initial concept of multistable plate structure with integral snap-through elements

analytically determined restoring force. A practical realization of this concept requires a specific bistable element that could actually be incorporated in a multistable plate. The starting point for our search for such an element was a recent study of multiconfiguration space frames [7] in which bistable struts based on the concept of the von Mises snap-through truss were used to form a space frame. The concept that was adopted in this study is shown in Fig. 5; it consists of two identical pairs of $5 \mathrm{~mm}$ wide and 0.35 $\mathrm{mm}$ thick plates attached to a stiff post and U-frame by means of "living hinges" [15]. This whole assembly was constructed as a single piece by injection molding Nylon6. These structures may be incorporated with actuators to enable remote transition. For example, a similar bistable snap-though structure has been demonstrated, which is successfully coupled with dielectric elastomer actuators to enable repeated back-and-forth transition [16].

The transition between the two configurations shown involves buckling of the plates, which must be sufficiently thin to avoid any permanent deformation during this process. The force-displacement of this structure has the simple trilinear form shown in Fig. 6 if the material behavior is idealized as linear-elastic and axial deformation is neglected. In reality, Nylon6 behaves as a viscoelastic solid at room temperature, and hence the measured force-displacement relationship is actually rate dependent; also, the axial stiffness of the plates is finite, and so the first and last parts of the response curve are not vertical.

The same concept was adopted for the present study; but, here, a single von Mises truss is sufficient for each bistable element because the compliant plate and corrugated plate structure assembly provide firm attachment points for the bistable elements. An initial implementation is shown in Figs. 7 and 8; note that the width of the U-frame has been set equal to the full width of the corrugated plate and the post has been shortened. Also, the bistable element has been connected to the corrugated plate structure by means of living hinges perpendicular to the plane of bending of the multistable structure, to allow the angle changes from $\theta_{1}$ to
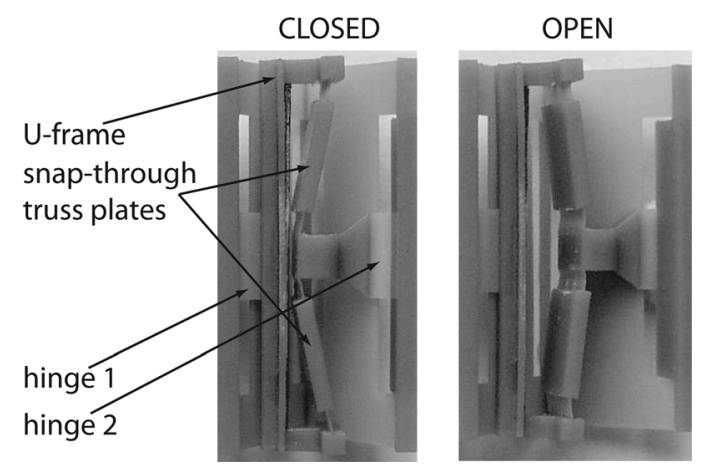

Fig. 8 Two configurations of bistable element in initial concept of multistable plate structure 


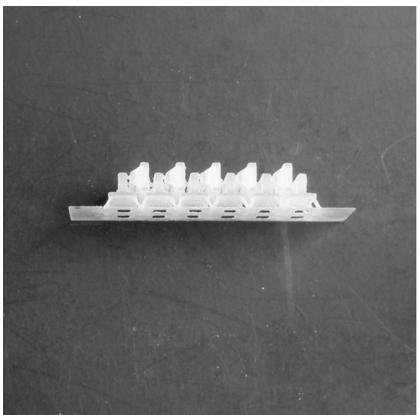

(a)

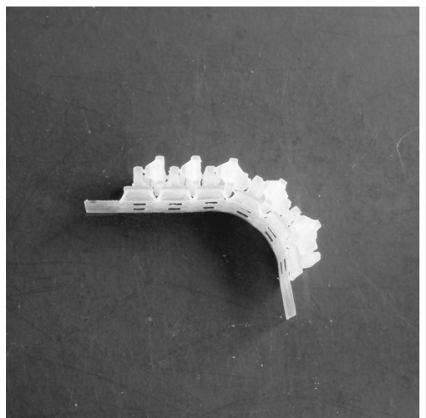

(d)

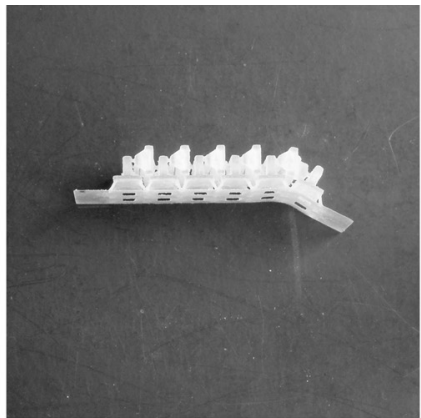

(b)

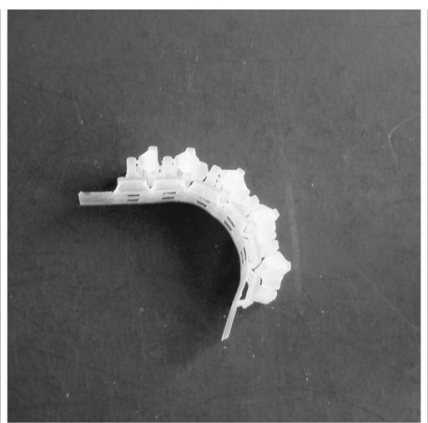

(e)

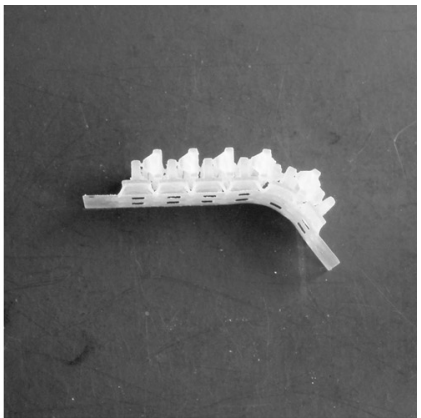

(c)

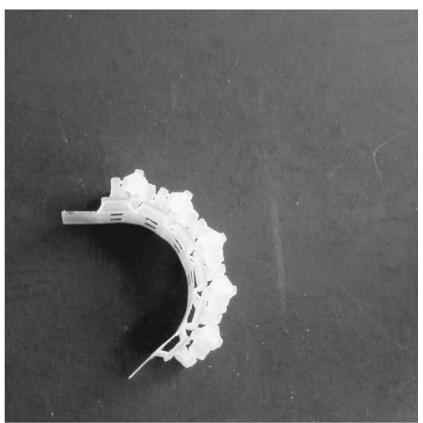

$(f)$

Fig. 9 Sequential actuation of prototype containing five bistable elements

$\theta_{2}$. This particular model was made by stereolithography with the material SI50 (also known as Accura 50).

There are several analytical solutions for the response of a simple von Mises truss [17,18], but they do not account for the effects of living hinges. Hence, a finite element simulation will be used in Sec. 5 to determine the force-displacement response of the present structure.

\section{Design and Realization of a Functional Prototype}

Our approach to verify the above analysis is to carry out a detailed analysis of the bistable element, which is the critical component of our design, as explained in Sec. 4, and to use the analytical tools of Sec. 3 to evaluate the rest of the design. The validity of this approach will then be demonstrated by constructing a functioning physical model. It was decided that performing a nonlinear finite element analysis of the complete system would not provide significant additional information.
A working prototype containing five bistable elements chained in sequence, resulting in a total of $2^{5}=32$ stable configurations, is shown in Fig. 9. The effect of sequential actuation of the five bistable elements is shown-each resulting in a different stable configuration. The length of the curved region increases in proportion to the number of elements that have been actuated.

This prototype was made by the PolyJet 3D printing rapid prototype technology [20] using the acrylic-based polymer FullCure720. This material has an unrelaxed modulus, evaluated using ASTM D-638 [21], of $2870 \mathrm{MPa}$ [22] and Poisson's ratio of 0.3 . This technology is advantageous for this application as it enables two materials - an acrylic-based photopolymer and a sacrificial support structure-to be deposited simultaneously, which allows complex three-dimensional structures to be made to high geometric accuracy.

A computer aided design model of this prototype is shown in Fig. 10. The design is monolithic as the bistable structures, the corrugated plate structure-based around equilateral triangles with side length $L=a=10 \mathrm{~mm}$ - and the compliant plate are all

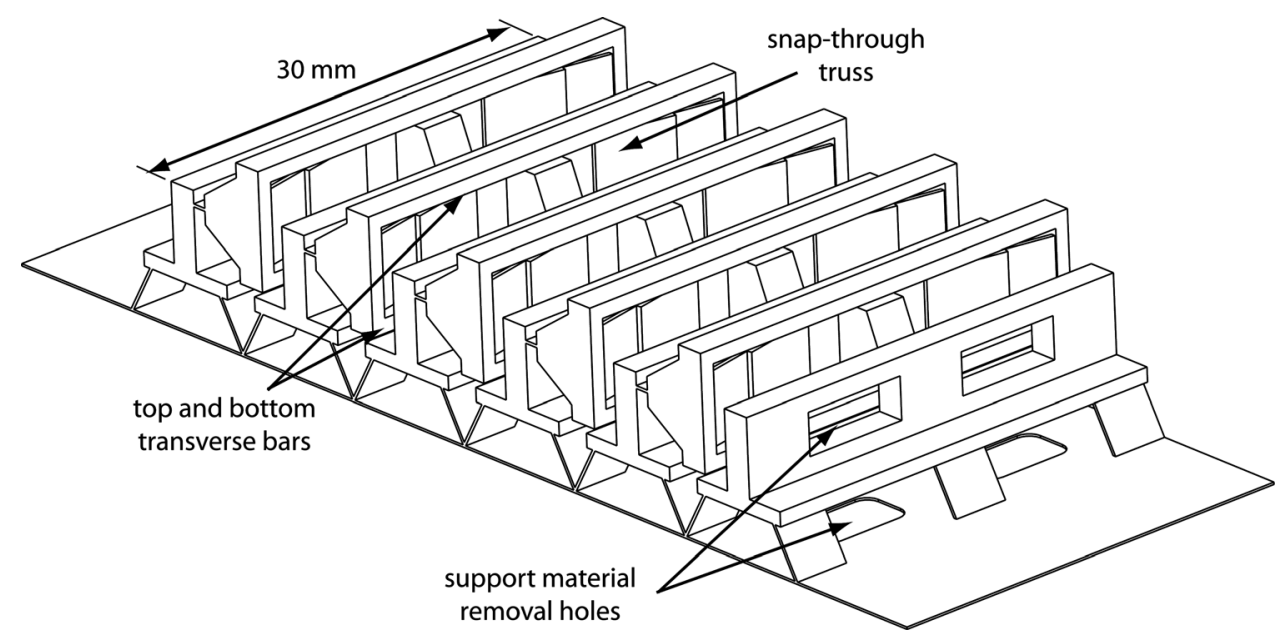

Fig. 10 Multistable plate structure prototype 


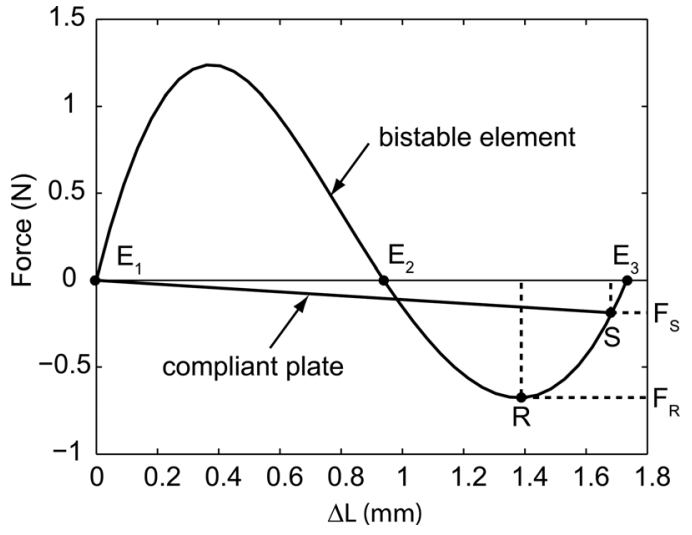

Fig. 11 Finite element simulation of force-displacement response of bistable element and restoring force exerted by compliant plate from Eq. (10)

fabricated together as a single entity. It can be seen that the Uframes have been stiffened by making them deeper and thicker, to ensure that the maximum snap-through force of the von Mises trusses is reached. Holes have been placed throughout the structure to facilitate the removal of the above-mentioned sacrificial support structure, which is deposited during fabrication.

In this design, the snap-through truss plates are $9.79 \mathrm{~mm}$ long, $9 \mathrm{~mm}$ wide, and $0.5 \mathrm{~mm}$ thick. They are connected to the rest of the bistable element by $2 \mathrm{~mm}$ long, $0.2 \mathrm{~mm}$ thick living hinges at either end.

FullCure720 is actually viscoelastic, but it is not necessary to consider time-dependence in the present design because the whole structure is made of the same material, and if a linear viscoelastic model is applicable [19], then all parts will relax at the same rate regardless of the initial level of strain. With these assumptions, the stability and locking criteria are dependent only on the geometry of the structure.

This design was verified by carrying out a finite-element simulation of the bistable element by itself, to capture the effects of the living hinges, which cannot be made by current rapid prototyping technologies as thin as by injection molding and, hence, provide a noticeable bias in the response curve toward the initially manufactured shape. The results of this analysis were then compared with the restoring behavior of the compliant plate and corrugated plate, already examined in Sec. 3 .

The displacement-controlled response of the bistable element was computed with a quasistatic nonlinear finite element analysis, using the commercial finite element software SAMCEF [23]. The structure was modeled with three-node Timoshenko beam elements. The results are shown in Fig. 11, where it can be seen that the bistable element is in equilibrium with zero external force in two different stable configurations, $\mathrm{E}_{1}$ and $\mathrm{E}_{3}$ : The displacement between these two configurations provides the stroke $\Delta L=1.73 \mathrm{~mm}$ of the bistable element. Note that there is also a third, intermediate equilibrium configuration, $\mathrm{E}_{2}$, which, however, is unstable.

Next, the restoring force due to the flexure of the $0.2 \mathrm{~mm}$ thick and $30 \mathrm{~mm}$ wide compliant plate was considered. The material removal holes reduce its effective width to $20 \mathrm{~mm}$. Taking the bistable element stroke $\Delta L$ as a variable, the restoring force can be evaluated using Eq. (10); this response is plotted with a dotted line in the figure. The intersection of the two response lines, marked with point $S$ in the figure, defines a stable equilibrium configuration for the whole structure. The corresponding stroke of the bistable element is $\Delta L=1.68 \mathrm{~mm}$, and hence the value of the peak curvature calculated from Eq. (9) is $\kappa_{p}=0.040 / \mathrm{mm}$.

The ratio between the force in the bistable element, $F_{S}$, and the maximum value, $F_{R}$, before the element snaps back is $F_{R} / F_{S}=3.3$.
Hence, a substantial margin against the restoring force exerted by the compliant plate causing snap-back is predicted. This result indicates that the plate is expected to lock in its curved configuration, and this behavior was indeed confirmed by the experimental verification shown in Fig. 9.

\section{Conclusion}

This paper has proposed and demonstrated a novel concept for a multistable structure that may be used to impart discrete smooth curvature changes to a compliant plate. The proposed concept may be realized as a monolithic compliant mechanism, and this has been demonstrated through the fabrication of such a structure using rapid-prototyping technology. Our designs of the demonstration models were initially formulated following the simple geometric approach presented in Sec. 3, based on a simple analytical estimate of the restoring force that must be sustained by the bistable elements in order to lock the plate in a curved configuration. Once a detailed design had been worked out, the analysis of the restoring force was refined by means of a full finite-element simulation that incorporated the effects of the living hinges in the structure.

Viscoelastic effects were neglected in the analysis as it was argued that, in a linear viscoelastic model, the stress distribution would decay at the same rate everywhere. This is a reasonable assumption in a structure made of the same material throughout, provided that time-dependent shape changes can be neglected. Of course, this aspect of the analysis could be refined.

The most challenging part of the work that has been presented in this paper has been the construction of functional demonstration models that would be useful in practical applications. Injection molding is likely to be a cost-effective approach for large scale manufacturing, as it enables the construction of robust living hinges, but the cost of tooling is prohibitive for initial verification models. After exploring a number of alternatives, the approach that was adopted for the models presented in this paper was to use acrylic-based laser-sintered photopolymers, which, however, are not suitable for an arduous operating environment and are prone to fatigue failure when subjected to repeated cyclic loading during the operation of the structure.

Several high-tech potential applications of the proposed concept have been identified in the introduction, but, in fact, it seems likely that the combination of multistability and compliant mechanisms will make our approach attractive for high volume consumer products. The application of curvature in one-dimension is of course useful for many such applications, but the ability to impart complex local three-dimensional shapes to an initially smooth surface may be even more desirable. Hence, an extension of the presented work to surfaces with nonzero Gaussian curvature should be considered.

\section{Acknowledgment}

The research presented in this paper was funded by the Cambridge-MIT Institute (CMI) and was carried out in the Department of Engineering, University of Cambridge. Alastair Ross provided assistance with the fabrication of the prototype. Tyge Schiøler is gratefully acknowledged for his assistance and involvement in the early stages of this research.

\section{References}

[1] Ogawa, H., Babinsky, H., Pätzold, M. and Lutz, T., 2008, "Shock/BoundaryLayer Interaction Control Using Three-Dimensional Bumps for Transonic Wings," AIAA J., 46, pp. 1442-1452.

2] Cambridge Enterprise Ltd., Santer, M., Pellegrino, S., and Schiøler, T., 2009 , "Reversibly Deformable Structure," UK Patent Application GB-2452089-A, Feb. 25, 2009.

[3] Mattioni, F., Weaver, P. M., Potter, K. D., and Friswell, M. I., 2008, “Analysis of Thermally Induced Multistable Composites,” Int. J. Solids Struct., 45, pp. $657-675$. 
[4] Iqbal, K., and Pellegrino, S., 2000, "Bi-Stable Composite Shells," Proceedings of the 41st AIAA ASME/ASCE/AHS/ASC Structures, Structural Dynamics and Materials Conference, AIAA Paper No. 2000-1385.

[5] Qiu, J., Lang, J. H., and Slocum, A. H., 2001, "A Centrally-Clamped ParallelBeam Bistable MEMS Mechanism," 14th IEEE International Conference on Micro Electro Mechanical Systems, pp. 353-356.

[6] Norman, A., Seffen, K., and Guest, S., 2009, "Morphing of Curved Corrugated Shells," Int. J. Solids Struct., 46, pp. 1624-1633.

[7] Schiøler, T., and Pellegrino, S., 2007, "Space Frames With Multiple Stable Configurations," AIAA J., 45, pp. 1740-1747.

[8] Santer, M., and Pellegrino, S., 2009, "Topological Optimization of Compliant Adaptive Wing Structure," AIAA J., 47, pp. 523-534.

[9] Lu, K.-J., and Kota, S., 2005, "An Effective Method of Synthesizing Compliant Adaptive Structures Using Load Path Representation,” J. Intell. Mater. Syst. Struct., 16, pp. 307-317.

[10] Lu, K.-J., and Kota, S., 2003, "Design of Compliant Mechanisms for Morphing Structural Shapes,” J. Intell. Mater. Syst. Struct., 14, pp. 379-391.

[11] Diaconu, C., Weaver, P., and Mattioni, F., 2008, "Concepts for Morphing Air foil Sections Using Bi-Stable Laminated Composite Structures," Thin-Walled Struct., 46, pp. 689-701.

[12] Chirikjian, G., 1994, "A binary paradigm for robotic manipulators," Proceedings of the 1994 IEEE International Conference on Robotics and Automation, San Diego, California, IEEE, pp. 3063-3069.

[13] dos Santos e Lucato, S. L., Wang, J., Maxwell, P., McMeeking, R. M., and Evans, A. G., 2004, "Design and Demonstration of a High Authority Shape Morphing Structure,” Int. J. Solids Struct., 41, pp. 3521-3543.
[14] Wicks, N., and Hutchinson, J., 2004, "Sandwich Plates Actuated by a Kagome Planar Truss,” ASME J. Mech. Des., 71, pp. 652-662.

[15] Howell, L., 2001, Compliant Mechanisms, 1st ed., John Wiley \& Sons, New York.

[16] Plante, J. S., Santer, M., Dubowsky, S., and Pellegrino, S., 2005, "Compliant Bistable Dielectric Elastomer Actuators for Binary Mechatronic Systems," Proceedings of the IDECT/CIE 2005: ASME Mechanism and Robotics Conference, pp. 121-126.

[17] Bažant, Z., and Cedolin, L., 1991, Stability of Structures. Elastic, Inelastic, Fracture and Damage Theories, 1 st ed., Oxford University, New York.

[18] Fichter, W., and Pinson, M., 1989, "Load-shortening behavior of an initially curved eccentrically loaded column," NASA Technical Memorandum 101643.

[19] Christensen, R., 2003 (reprint), Theory of Viscoelasticity, 2nd ed., Dover Publications, New York.

[20] PolyJet Matrix Technology, 2009, Object Geometries, Ltd., Holtzman St., Science Park, P.O. Box 2496, Rehovot 76124, Israel. URL http://www.objet.com/ docs/PolyJet\%20Matrix\%203D\%20Printing\%20Technology\%20A4.pdf (accessed July 31, 2009).

[21] ASTM Standard 638, 2008, Standard Test Method for Tensile Properties of Plastics (ASTM International, West Conshohocken, PA), DOI:10.1520/D0638-08.

[22] FullCure Materials, 2009, Objet Geometries, Ltd., Holtzman St., Science Park, P.O. Box 2496, Rehovot 76124, Israel. URL http://www.objet.com/docs/FullCure 3D\%20Modeling_A4_il.pdf (accessed July 31, 2009).

[23] SAMCEF v12.0-03 Finite Element Package, Samtech S.A., Rue des ChasseursArdennais, 8B-4031 Liège (Angleur), Belgium. 